\title{
Numerical Solution of Mixed Problem of Parabolic Equation with an Integral Conditions by using Finite Difference and Orthogonal Function Approximation
}

\author{
Oussaeif Taki-Eddine*, Bouziani Abdelfatah \\ AND NAOUi GATTAL
}

\begin{abstract}
In this work the combined finite difference and orthogonal function approximation methods have been proposed for the numerical solution of the one-dimensional parabolic equation with two integrals conditions. The time variable is approximated using a finite difference scheme. But the orthogonal function approximation is employed for discretizing the space variable by using orthogonal cosine function.
\end{abstract}

\section{INTRODUCTION}

In the rectangle $\Omega=(0, \beta) \times(0, T)$, with $T<\infty$, we consider the following problem

$$
\begin{gathered}
\mathcal{L} v=v_{t}-\left(a(x, t) v_{x}\right)_{x}+p(x, t) v_{x}+c(x, t) v=E(x, t), \\
0<x<\beta, \quad 0<t<T,
\end{gathered}
$$

where the functions $a(x, t), p(x, t)$ and $c(x, t)$ and their first derivatives are bounded in $\Omega$.

We adjoin to equation (1.1) the initial condition

$$
\ell v=v(x, 0)=\phi(x), \quad x \in(0, \beta),
$$

with integral conditions

$$
\begin{aligned}
& \int_{0}^{\beta} v(x, t) \mathrm{d} x=\psi(t), \\
& \int_{0}^{\beta} x v(x, t) \mathrm{d} x=\chi(t),
\end{aligned}
$$

where $\phi, \psi, \chi, a, p, E$ are known functions, and $\beta$ and $T$ are given constants.

Key words and phrases. Parabolic Equation; Finite Difference; Orthogonal Function; Spectral Methods; Mixed Problem; Integral Condition.

${ }^{*}$ Corresponding author. 
We shall assume that the function $\phi$ satisfies the following compatibility conditions:

$$
\begin{gathered}
\int_{0}^{\beta} \phi(x) \mathrm{d} x=\psi(0), \\
\int_{0}^{\beta} x \phi(x) \mathrm{d} x=\chi(0) .
\end{gathered}
$$

The mathematical modeling of this type of problems are encountered in heat transmission theory, in thermoelasticity, and in plasma physics $[2,3]$ and can be reduced to the nonlocal problems. Therefor, partial differential equations with nonlocal boundary conditions have received much attention in last 20 years. However, most of the articles were directed to the second order parabolic equations, particularly to heat conduction equations.

The parabolic equation with nonlocal boundary conditions has been treated extensively by finite difference methods, finite element procedures, boundary element techniques, spectral schemes, Adomian decomposition method, and the semidiscretization procedures in the last 20 years $[5,6,7,8]$. We will deal here with new type of nonlocal boundary value problems, Dehgan [9] investigated this type of problems and presented serval finite difference schemes for numerical solution of hyperbolic equations arising boundary value problems with integral condition. The numerical techniques developed in [9] are based on three-level explicit finite difference procedures. Ang [10] developed a numerical technique for solution of the studied model. His scheme is based on integro-differential equation and local interpolating functions for solving the one-dimensional wave equation subject to a nonlocal conservation condition and suitably prescribed initial boundary conditions, but in [4] a different approach is used by using combined finite difference and spectral methods for solving the hyperbolic equation with integral condition. The proof of the existence, uniqueness and continuous dependence of the strong solution upon the data for an initial-boundary value problem and integral conditions for this problem is studied by Bouziani [1].

The rest of this paper is organized as follows, in the next section, reformulation of the problem for facilitate the study. The finite difference scheme and spectral methods are introduced in Section 3. The finite difference scheme and orthogonal function approximation are applied to problem (1.1)-(1.4) in Section 4. The numerical example are presented in Section 5. Section 6 ends this paper with a brief conclusion.

\section{ReFormulation of the PRoblem}

Since the integral conditions are nonhomogeneous, we reduce the nonhomogeneous boundary conditions to homogeneous conditions, by introducing a new, unknown function $u$ defined as:

$$
v(x, t)=u(x, t)+K(x, t),
$$


where

$$
K(x, t)=\frac{1}{\beta} \psi(t)+\frac{6}{\beta^{4}}\left(3 x^{2}-2 \beta x\right)[2 \chi(t)-\beta \psi(t)] .
$$

Then the function $u(x, t)$ has to satisfy the equation $\mathcal{L} u=f-\mathcal{L} K$ with the corresponding homogeneous integral conditions.

Therefore problem (1.1)-(1.4) is equivalent to the following:

$$
\mathcal{L} u=E-\mathcal{L} K=f(x, t), \quad 0<x<\beta, 0<t<T,
$$

with initial condition

$$
\ell u=u(x, 0)=\phi(x)-\ell K=\varphi(x), \quad x \in(0, \beta),
$$

integral conditions

$$
\begin{array}{r}
\int_{0}^{\beta} u(x, t) \mathrm{d} x=0, \\
\int_{0}^{\beta} x u(x, t) \mathrm{d} x=0,
\end{array}
$$

and the following compatibility conditions:

$$
\begin{gathered}
\int_{0}^{\beta} \varphi(x) \mathrm{d} x=0, \\
\int_{0}^{\beta} x \varphi(x) \mathrm{d} x=0 .
\end{gathered}
$$

\section{Analysis of Spectral Methods}

The spectral methods $[11,12,13]$ for a function $U(x)$, square integrable in $[0, \beta)$, may be approximated by another function by using orthogonal function (OFs) as:

$$
U(x)=\sum_{k=0}^{\infty} d_{k} H_{k}(x),
$$

where $\left\{H_{0}(x), H_{1}(x), H_{2}(x), \ldots, H_{M}(x), \ldots\right\}$ be an orthogonal set of functions on the interval $[0, \beta]$ :

$$
\int_{0}^{\beta} H_{k}(x) H_{i}(x) \mathrm{d} x= \begin{cases}0, & \text { when } k \neq i, \\ \left\|H_{k}\right\|, & \text { when } k=i,\end{cases}
$$

and $d_{k}$ is the corresponding coefficient. But in practice we need only the first $(M+1)$ term OFs, then we have

$$
U(x)=\sum_{k=0}^{M} d_{k} H_{k}(x) .
$$


These parameters $d_{k}$ must be chosen in such a way that the approximation is "as good as possible" in the sense to minimize the mean integral square error

$$
\varepsilon=\int_{0}^{\beta}\left(U(x)-\sum_{k=0}^{M} d_{k} H_{k}(x)\right)^{2} \mathrm{~d} x .
$$

The expression of $d_{k}$ was derived by minimizing $\varepsilon$ by means of derivatives with respect to every $d_{k}, k=0,1,2, \ldots, M$, so we obtain:

$$
d_{k}=\frac{1}{\left\|H_{k}\right\|} \int_{0}^{\beta} U(x) H_{k}(x) \mathrm{d} x .
$$

\section{Resolution of the Problem hith Combined Finite} Difference and Orthogonal Function Approximation

We start by dividing only the domain $[0, T]$ into an $N$ subintervals in $t$ direction, and the time step size $h=\frac{T}{N}$. The points $\left(t_{j}\right)$ are defined by

$$
t_{j}=j h, \quad j=0,1,2, \ldots, N .
$$

According to integral conditions (2.5), we take

$$
H_{k}(x)=\cos \frac{2 k \pi x}{\beta}, \quad \forall x \in[0, \beta] .
$$

So, we give

$$
u_{M}(x, t) \simeq \sum_{k=0}^{M} d_{k}(t) \cos \frac{2 k \pi x}{\beta} .
$$

Using a forward difference scheme at time $\left(t_{j}\right)$ we get the recurrence equation:

$$
\frac{u^{j+1}-u^{j}}{h}=\frac{\partial}{\partial x}\left(a^{j} \frac{\partial}{\partial x} u^{j}\right)-p^{j} \frac{\partial}{\partial x} u^{j}-c^{j} u^{j}+f^{j}, \quad 0 \leq j \leq N,
$$

where $u^{j}=u\left(x, t_{j}\right), a^{j}=a\left(x, t_{j}\right), p^{j}=p\left(x, t_{j}\right), c^{j}=c\left(x, t_{j}\right)$ and $f^{j}=$ $f\left(x, t_{j}\right)$.

By using the spectral-Galerkin method, we get

$$
\begin{aligned}
& \left\langle\frac{u^{j+1}-u^{j}}{h}, \cos \frac{2 k \pi}{\beta}\right\rangle \\
= & \left\langle\frac{\partial}{\partial x}\left(a^{j} \frac{\partial}{\partial x} u^{j}\right)-p^{j} \frac{\partial}{\partial x} u^{j}-c^{j} u^{j}+f^{j}, \cos \frac{2 k \pi}{\beta}\right\rangle, \quad 0 \leq k \leq M,
\end{aligned}
$$

where the inner product of two function $g$ and $z$ on interval $[0, \beta]$ are defined by

$$
\langle g, z\rangle=\int_{0}^{l} w(x) z(x) g(x) \mathrm{d} x,
$$

where $w(x)$ is the weight function. 
By the orthogonality of cosine functions on interval $[0, l]$, we obtain

$$
\begin{aligned}
& \left\langle\frac{u^{j+1}-u^{j}}{h}, \cos \frac{2 k \pi}{\beta}\right\rangle \\
= & -\left\langle\left(a^{j} \frac{\partial}{\partial x} u^{j}\right), \frac{\partial}{\partial x} \cos \frac{2 k \pi}{\beta}\right\rangle \\
& -\left\langle p^{j} \frac{\partial}{\partial x} u^{j}+c^{j} u^{j}-f^{j}, \cos \frac{2 k \pi}{\beta}\right\rangle \\
= & -\left\langle\frac{2 k \pi}{\beta} u^{j},\left(a_{x}^{j} \sin \frac{2 k \pi}{\beta}\right)+\left(\frac{2 k \pi}{\beta} a^{j} \cos \frac{2 k \pi}{\beta}\right)\right\rangle \\
& -\left\langle p^{j} \frac{\partial}{\partial x} u^{j}+c^{j} u^{j}-f^{j}, \cos \frac{2 k \pi}{\beta}\right\rangle \\
= & -\frac{4 k^{2} \pi^{2}}{\beta^{2}}\left\langle u^{j}, a^{j} \cos \frac{2 k \pi}{\beta}\right\rangle-\frac{2 k \pi}{\beta}\left\langle u^{j}, a_{x}^{j} \sin \frac{2 k \pi}{\beta}\right\rangle \\
& -\left\langle p^{j} \frac{\partial}{\partial x} u^{j}+c^{j} u^{j}-f^{j}, \cos \frac{2 k \pi}{\beta}\right\rangle,
\end{aligned}
$$

where $a_{x}^{j}=\frac{\partial}{\partial x} a\left(x, t_{j}\right)$.

With respect to the weight function $w(x)=1$, and by using the properties of cosine and sine functions, we have:

$$
\begin{aligned}
\frac{d_{k}^{j+1}-d_{k}^{j}}{h}= & -\left[\frac{4 k^{2} \pi^{2}}{\beta^{2}} d_{k}^{j} \int_{0}^{\beta} a\left(x, t_{j}\right) \cos ^{2} \frac{2 k \pi x}{\beta} \mathrm{d} x\right] \\
& -\left[\frac{2 k \pi}{\beta} d_{k}^{j} \int_{0}^{\beta}\left(\frac{\partial}{\partial x} a\left(x, t_{j}\right)\right) \cos \frac{2 k \pi x}{\beta} \sin \frac{2 k \pi x}{\beta} \mathrm{d} x\right] \\
& -\left[\frac{2 k \pi}{\beta} d_{k}^{j} \int_{0}^{\beta} p\left(x, t_{j}\right) \cos \frac{2 k \pi x}{\beta} \sin \frac{2 k \pi x}{\beta} \mathrm{d} x\right] \\
& -\left[d_{k}^{j} \int_{0}^{\beta} c\left(x, t_{j}\right) \cos ^{2} \frac{2 k \pi x}{\beta} \mathrm{d} x\right] \\
& +\left[\int_{0}^{\beta} f\left(x, t_{j}\right) \cos \frac{2 k \pi x}{\beta} \mathrm{d} x\right] .
\end{aligned}
$$


Then, we obtain:

$$
\begin{aligned}
\frac{d_{k}^{j+1}-d_{k}^{j}}{h}= & -\left[\frac{2 k^{2} \pi^{2}}{\beta^{2}} d_{k}^{j} \int_{0}^{\beta} a\left(x, t_{j}\right)\left(1+\cos \frac{4 k \pi x}{\beta}\right) \mathrm{d} x\right] \\
& -\left[\frac{k \pi}{\beta} d_{k}^{j} \int_{0}^{\beta}\left(\frac{\partial}{\partial x} a\left(x, t_{j}\right)\right) \sin \frac{4 k \pi x}{\beta} \mathrm{d} x\right] \\
& -\left[\frac{k \pi}{\beta} d_{k}^{j} \int_{0}^{\beta} p\left(x, t_{j}\right) \sin \frac{4 k \pi x}{\beta} \mathrm{d} x\right] \\
& -\left[\frac{1}{2} d_{k}^{j} \int_{0}^{\beta} c\left(x, t_{j}\right)\left(1+\cos \frac{4 k \pi x}{\beta}\right) \mathrm{d} x\right] \\
& +\left[\int_{0}^{\beta} f\left(x, t_{j}\right) \cos \frac{2 k \pi x}{\beta} \mathrm{d} x\right] .
\end{aligned}
$$

We put:

$$
\begin{gathered}
\int_{0}^{\beta} a\left(x, t_{j}\right)\left(1+\cos \frac{4 k \pi x}{\beta}\right) \mathrm{d} x=A_{k}^{j}, \\
\int_{0}^{\beta}\left(\frac{\partial}{\partial x} a\left(x, t_{j}\right)\right) \sin \frac{4 k \pi x}{\beta} \mathrm{d} x=A_{k, x}^{j}, \\
\int_{0}^{\beta} p\left(x, t_{j}\right) \sin \frac{4 k \pi x}{\beta} \mathrm{d} x=P_{k}^{j}, \\
\int_{0}^{\beta} c\left(x, t_{j}\right)\left(1+\cos \frac{4 k \pi x}{\beta}\right) \mathrm{d} x=C_{k}^{j}, \\
\int_{0}^{\beta} f\left(x, t_{j}\right) \cos \frac{2 k \pi x}{\beta} \mathrm{d} x=F_{k}^{j} .
\end{gathered}
$$


Then, we obtain

$$
\frac{d_{k}^{j+1}-d_{k}^{j}}{h}=-\frac{2 k^{2} \pi^{2}}{\beta^{2}} d_{k}^{j} A_{k}^{j}-\frac{k \pi}{\beta} d_{k}^{j} A_{k, x}^{j}-\frac{k \pi}{\beta} d_{k}^{j} P_{k}^{j}-\frac{1}{2} d_{k}^{j} C_{k}^{j}+F_{k}^{j} .
$$

Similarly by using the initial condition, we obtain:

$$
\left\langle u_{k}^{0}, \cos \frac{2 k \pi}{\beta}\right\rangle=\left\langle\varphi, \cos \frac{2 k \pi}{\beta}\right\rangle, \quad 0 \leq k \leq M,
$$

and by orthogonality property we obtain:

$$
d_{k}^{0}=\varphi_{k}=\int_{0}^{\beta} \varphi(x) \cos \frac{2 k \pi x}{\beta} \mathrm{d} x, \quad 0 \leq k \leq M .
$$

The values $\varphi_{k}, F_{k}^{j}, A_{k}^{j}, A_{k, x}^{j}, P_{k}^{j}, C_{k}^{j}$ are the Fourier coefficient of $\varphi(x)$, $f(x), \ldots, C\left(x_{k}, t_{j}\right)$. Finally, we get:

$$
\begin{aligned}
d_{k}^{j+1}= & \left(1-\frac{2 h k^{2} \pi^{2}}{\beta^{2}} A_{k}^{j}-\frac{h k \pi}{\beta} A_{k, x}^{j}-\frac{h k \pi}{\beta} P_{k}^{j}-\frac{h}{2} C_{k}^{j}\right) d_{k}^{j}+h F_{k}^{j} \\
& \text { for } 1 \leq j \leq N, 0 \leq k \leq M .
\end{aligned}
$$

Therefore we can evaluate the coefficient $d_{k}^{j}$.

\section{Numerical EXAmple}

In this section, we present and discuss the numerical results by employing Finite Difference and orthogonal function approximation for one test example.

Consider the following equation

$$
\mathcal{L} u=u_{t}+\left(\frac{1}{(t+1) 4 \pi^{2}} u_{x}\right)_{x}=0, \quad 0<x<1, \quad 0<t<1,
$$

with the initial condition

$$
\varphi(x)=\cos 2 \pi x, \quad x \in(0,1),
$$

and with the integral conditions

$$
\begin{aligned}
& \int_{0}^{1} u(x, t) \mathrm{d} x=0, \\
& \int_{0}^{1} x u(x, t) \mathrm{d} x=0,
\end{aligned} \quad t \in(0,1.0),
$$

which is easily seen to have the exact solution

$$
u(x, t)=(t+1) \cos 2 \pi x .
$$

The result with $h=\frac{1}{20}$ and $M=10$, using the finite difference scheme and spectral methods by using orthogonal function approximation based on the preceding sections, are shown in Table 1 . In this table, we present and provide a comparison between exact solution and the solution of the new 
technique described in this paper for time step 0.15, then the comparison is for $u(x, 0.15)$. So, we have

$$
d_{k}^{j+1}=\left(1-2 h k^{2} \pi^{2} A_{k}^{j}\right) d_{k}^{j},
$$

where

$$
A_{k}^{j}=\frac{-1}{\left(t_{j}+1\right) 4 \pi^{2}} .
$$

Then, we obtain

$$
d_{k}^{j+1}=\left(1+\frac{h k^{2}}{2\left(t_{j}+1\right)}\right) d_{k}^{j} .
$$

Note that the results obtained by using our methods (Finite Difference and Orthogonal Function Approximation) are more accurate to exact results, but for good results you must divide the space for more step, and chose the time step size very small, so we obtained good results, which indicate in Table 1.

TABLE 1

\begin{tabular}{|c|c|c|}
\hline Step $x$ & Exact $u$ & $\begin{array}{c}\text { Finite Difference and } \\
\text { orthogonal function } \\
\text { approximate }\end{array}$ \\
\hline \hline 0.1 & 1.1499308522642 & 1.1499308522542 \\
0.2 & 1.1497234173721 & 1.1497234173621 \\
0.3 & 1.1493777202693 & 1.1493777202593 \\
0.4 & 1.1488938025283 & 1.1488938025183 \\
0.5 & 1.1482717223434 & 1.1482717223335 \\
0.6 & 1.1475115545243 & 1.1475115545143 \\
0.7 & 1.1466133904862 & 1.1466133904763 \\
0.8 & 1.1455773382398 & 1.1455773382298 \\
0.9 & 1.1444035223773 & 1.1444035223674 \\
1.0 & 1.1430920840584 & 1.1430920840485 \\
\hline
\end{tabular}




\section{Conclusion}

In this paper, the finite difference and orthogonal function approximation was employed successfully for solving the mixed problem of parabolic equation with integrals conditions. This method solves the problem by approximated the time variable by using a finite difference scheme and but the orthogonal function approximation is employed for discretizing the space variable by using orthogonal cosine function. The numerical results show that our new technique described in this paper is an accurate and reliable analytical technique worked very well for the studied one-dimensional parabolic equation with integrals conditions. The new technique can be extended to two-dimensional parabolic equation with integral conditions, also, can be used other orthogonal polynomial for the approximation.

\section{REFERENCES}

[1] A. Bouziani and N.E. Benouar, Problème mixte avec conditions intégrales pour une classe d'équations paraboliques, Comptes Rendus de l'Académie des Sciences, Paris, t.321, Série I, (1995), 1177-1182.

[2] A.A. Samarskii, Some problems in differential equations theory, Differents. Uravn., 16 (1980), 1925-1935.

[3] P. Shi, M. Shilor, Design of contact patterns in one dimensional thermoelasticity, in Theorical Aspects of industrial design, Society of Industrial and Appl Math, Philadelphia 1992.

[4] M. Ramezani, M. Dehghan, M. Razzaghi, Combined Finite Difference and Spectral Methods for the Numerical Solution of Hyperbolic Equation with an Integral Condition, Numerical Methods for Partial Differential Equations, 24 (2007), 1-8.

[5] M. Dehghan, Numerical solution of a parabolic equation with non-local boundary specifications, Appl. Math. Computat., 145 (2003), 185-194.

[6] M. Dehghan, The use of Adomian decomposition method for solving the one dimentional parabolic equation with non-local boundary spesification, Int. J. Comput. Math., 81 (2004), 25-34.

[7] M. Dehghan, Efficient techniques for the second-order parabolic equation subject to nonlocal specifications, Appl. Numer. Math., 52 (2005), 39-62.

[8] M. Dehghan, Solution of partial integro-differential equation arising from viscoelasticity, Int. J. Comput. Math., 83 (2006), 123-129.

[9] M. Dehghan, On the solution of an initial-boundary value problem that combines Neumann and integral condition for the wave equation, Numer. Methods Partial Differential Equations, 21 (2005), 24-40.

[10] W. T. Ang, A numerical method for the wave equation subject to a non-local conservation condition, Appl. Numer. Math., 56 (2006), 1054-1060.

[11] C. Hwang and Y. P. Shih, Laguerre series direct method for variational problems, J. Optim. Theory Applic., 39 (1983), 143-149. 
[12] R. Y. Chang and M. L. Wang, Shifted Legendre function approximation of differential equations; application to crystallization processes, Comput. Chem. Eng., 8 (1984), $117-125$.

[13] I. R. Horng and J. H. Chou, Shifted Chebyshev direct method for solving variational problems, Int. J. Syst. Sci., 16 (1985), 855-861.

[14] A. Bouziani, On the solvability of parabolic and hyperbolic problems with a boundary integral condition, International J. of Math and Math Sciences, Vol. 31, Issue 4 (2002), 201-213.

\section{Oussaeif TAKI-Eddine}

Department of Mathematics and Informatics The Larbi Ben M'hidi University

Oum El Bouagui B.P. 565

04000 Algeria

E-mail address: taki_maths@live.fr

\section{Bouziani Abdelfatah}

Department of Mathematics and Informatics The Larbi Ben M'hidi University

Oum El Bouagui B.P. 565

04000 Algeria

E-mail address: af_bouziani@hotmail.com

\section{Naoui Gattal}

Depatment of Mathematics and Informatics Cheikh El Arbi Tébessi University 12002 TÉBESSA

Algeria

E-mail address: naoui.gattal@gmail.com 